\title{
¿A qué llamamos violencia en las ciencias sociales?*
}

\author{
Santiago Álvarez
}

Recibido: 3 de septiembre de 2012

Evaluado: 18 de noviembre de 2012

Aceptado: 7 de febrero de 2013

\section{RESUMEN}

Este artículo se propone reflexionar sobre la ambigüedad del término violencia y la practicidad de su utilización en el campo de las ciencias sociales. Además, expone sus principales acepciones, tratando de pensar sobre las consecuencias que conlleva elegir cada una de ellas; debate la utilidad del uso del concepto teniendo en cuenta su evidente polisemia; y discute la posibilidad de aceptar una definición transcultural mínima. Por último, trata de responder a la pregunta sobre cuál concepto es el más adecuado para ser utilizado como herramienta en la investigación del conflicto político colombiano.

Palabras clave: violencia, conflicto, agresión, género, violencia simbólica, violencia ilegítima.

** Doctor en Antropología por la London School of Economics and Political. Actualmente forma parte del Centro de Antropología del Instituto de Desarrollo Económico y Social y es profesor de la Maestría en Antropología Social IDES-IDAES de las Universidad San Martín. Correo electrónico: alvaresantiago@hotmail.com 


\section{What do we call violence in the social sciences?}

\section{Abstract}

This paper aims at reflecting on the ambiguity of the term violence and its practical use in the field of social sciences. It also exposes its main meanings trying to think about the conAceptado: 7 de febrero de 2013 sequences that choosing any of them entails, debates on the usefulness of the concept given its apparent polysemy and discusses the possibility of accepting a minimum transcultural definition. Finally, it attempts to answer the question about which concept is best suited for use as a research tool in the Colombian political conflict.

Keywords: violence, conflict, aggression, gender, symbolic violence, unlawful violence. 


\section{¿A o que chamamos de violência nas ciências sociais?}

Recibido: 3 de septiembre de 2012
Evaluado: 18 de noviembre de 2012
Aceptado: 7 de febrero de 2013

\section{Resumo}

Este artigo tem como objetivo refletir sobre a ambiguidade do termo violência e a praticidade de sua utilização no campo das ciências sociais. Além do mais expõe suas principais acepções, tentando pensar sob as consequências que isso implica na escolha de cada uma delas; debate a utilidade do uso do conceito tendo em conta sua evidente polissemia; e discute a possibilidade de aceitar uma definição transcultural mínima. Finalmente, tenta responder à pergunta de qual conceito é mais adequado para ser usado como uma ferramenta na pesquisa do conflito político colombiano.

Palabra-clave: violência, conflito, agressão, gênero, violência simbólica, violência ilegítima. 


\section{LOS PROBLEMAS QUE CONLLEVA EL CONCEPTO DE VIOLENCIA}

Evidentemente, la palabra violencia es polisémica y ambigua, lo cual ha llevado a algunos autores a preguntarse sobre su utilidad. Dentro de esta postura, Heelas (1982) considera que el de la violencia no es un concepto sociológicamente útil y que no puede usarse una definición de este fuera del contexto cultural en que se origina.

Como Moore (1994) puntualiza, "a pesar de la enorme masa de escritura, investigación y especulación, el concepto de violencia para las ciencias sociales todavía continúa increíblemente subteorizado"1 (p. 138). Esto también se podría relacionar con una tendencia a incorporar al concepto de violencia a una serie de fenómenos cada vez más amplios, con el propósito de abarcar, por ejemplo, las desigualdades sociales, políticas y económicas. Por su parte, Rifiotis y Castelnuovo (2011) plantean:

\section{El término [violencia] se transformó así} en un significante vacío, un artefacto capaz de condensar múltiples significados y situaciones. Es que su campo semántico tiene una regla deformativa: la constante expansión. La aparente unidad del término resulta de una generalización implícita de los diversos fenómenos que ella designa siempre de modo homogeneizador, exterior y negativo (p. 12).

A su vez, estos autores sugieren que es más adecuado hablar de violencias, en plural:
Así, podemos afirmar que los discursos de la modernidad son insatisfactorios para la significación del mundo contemporáneo, tornándose entonces necesario realizar una revisión de los conceptos y las prácticas en el campo de "las violencias". Desde nuestro punto de vista, referirnos al término de modo plural significa afirmar la heterogeneidad y no circunscribirse a una tipología (Rifiotis y Castelnuovo, 2011, p. 12).

La expansión del término ha llevado a Garriga y Noel (2010) a hablar de la inflación del concepto de violencia:

En este sentido, nuestra elección del término inflación no es casual: sabemos que lo que un concepto gana en alcance, lo pierde en poder explicativo. Si un mismo término puede ser aplicado a tantos fenómenos y en ámbitos tan disímiles - desde, digamos, un insulto hasta un ataque terrorista-, podemos legítimamente preguntarnos en qué consistiría su utilidad. Si la violencia está en todas partes y predica de casi cualquier relación social concebible, ¿cuáles son las ventajas analíticas de utilizar un término con un referente tan vasto y difuso a la hora de pensar rigurosamente una $u$ otra variante de los fenómenos designados por él?

Tampoco puede usarse el concepto de violencia para describir toda acción social que implique contacto físico o comunicación verbal.

1 Traducción propia. 
Algunas feministas planteaban que la penetración sexual, cualquiera que fuera, era en sí misma violenta. Al respecto, Allison y Kilmartin (2007) consideran:

La violencia basada en género es todo ataque contra una persona (usualmente una mujer) como consecuencia, al menos en parte, de su posición desventajosa dentro de un sistema social dominado por el hombre².

Sería, si se llega a estos extremos, un concepto que no ayudaría mucho a iluminar los fenómenos que buscamos analizar. Estos intentos recuerdan lo que decía Hegel sobre el concepto de absoluto de Schelling: "Es como la noche en la que todos los gatos son pardos".

\section{LA VIOLENCIA SIMBÓLICA}

El concepto de Bourdieu y Passeron (1988) de violencia simbólica habla de formas de violencia no ejercidas por la fuerza física. Se trataría de la imposición, por parte de sujetos dominantes a los sujetos dominados, de una cosmovisión, roles sociales específicos, categorías cognitivas y estructuras mentales. Los autores hacen referencia específicamente al sistema educativo. En este sentido, Garriga y Noel (2010) plantean:

Esta noción de violencia simbólica se asemeja bastante a una serie de concepciones de inspiración más o menos psicoanalítica que piensan y entienden la socialización como una forma de violencia, en la medida en que la misma implica una imposición de un arbitrario cultural sobre una serie de impulsos pre- o parasociales ${ }^{3}$.

En este sentido, la violencia simbólica es fácilmente relacionable con el concepto marxiano de dominación, por la que los sujetos dominados reproducen y afianzan su sojuzgamiento. La dominación sería la probabilidad de encontrar obediencia sobre un grupo social específico. En toda relación de autoridad existe una voluntad de obediencia, una aceptación de la autoridad que se ejerce. Dado que ya existe un concepto, el de dominación, al que hacer referencia, ¿vale la pena mezclar ambos, produciendo una mayor confusión? En cambio, el de terror es un concepto específico claramente enriquecedor, diferente pero cercano al de violencia. Taussig usa el concepto de terror para hacer referencia a un poderoso discurso de dominación que actúa en la imaginación y los miedos de las personas. El terror, para él, es efectivo en "destruir la capacidad de resistencia de las personas" (Taussig, 1987, p. $128 ; 1992$, p. 11).

\section{LAS POSIBILIDADES DE CONSTRUIR UN CONCEPTO TRANSCULTURAL DE VIOLENCIA}

Además de una "inflación" del término, otro problema es la transculturalidad de la violencia, la posibilidad de establecer un concepto válido aplicable a diversas culturas: lo que para unos puede ser considerado violento, para otros no es percibido como tal; por ejemplo, ciertos juegos masculinos

3 Garriga y Noel (2010) alarman sobre la difusión y el uso, "a menudo salvaje", de la obra de Bourdieu.

2 Traducción propia. 
en los que los miembros de un grupo social se golpean hasta sangrar. Entre los nuer, los niños suelen pelearse usando brazaletes con púas (Pritchard, 1977). Situaciones que podrían denominarse de violencia doméstica entre el marido y su mujer no son percibidas del mismo modo en comunidades indígenas de los Andes, de acuerdo con los estudios realizados por Harris (1994) en Bolivia y por Harvey (1994) en Perú. Además, la violencia en estos casos es percibida no como disruptiva, sino como constitutiva de las relaciones sociales familiares.

Riches (1988) muestra cómo en el mundo anglosajón la violencia está siempre relacionada con la ilegitimidad. Así, si un grupo de policías estuviera ejerciendo una deliberada fuerza física sobre otros, dentro de las pautas y protocolos legales, su accionar no sería percibido como violento. Afirma el autor: "Cuando un testigo o una víctima invoca la noción de violencia, emite un juicio no solo de que la acción requerida causa daño físico, sino también de que es ilegítima" (Riches, 1988, p. 18). Por el contrario, Benjamin (2010, p. 87), reflexionando sobre el estado totalitario nacionalsocialista alemán, analiza críticamente la relación de la violencia con el derecho y la justicia. Plantea la doble cara de Jano del Estado, compuesta, por un lado, de razón y, por el otro, de violencia. El Estado expresaría dramáticamente esta contradicción en el caso del estado de emergencia ${ }^{4}$. Nada de esto está presente en el concepto anglosajón, en el que las

4 Taussig (1999) hace referencia a esta contradicción presente en la definición de Weber, quien consideraba al monopolio del uso legítimo de la fuerza dentro de un territorio determinado como la componente crucial del Estado: "Lo que debemos destacar aquí es cómo esta conjunción de violencia y razón es tan evidente y al mismo tiempo tan negada" (Taussig, 1999, p. 149). funciones en las que el Estado ejerce la fuerza no son percibidas críticamente.

Riches (1988) señala otras características emic de la violencia dentro de la misma cultura anglosajona: la violencia dentro del mundo anglosajón es asociada con inaceptabilidad, irracionalidad y bestialidad (pp. 18-20). Esta asociación con los animales es también típica, al menos en la Argentina, en los comentarios de los medios de comunicación deportiva con respecto a las hinchadas de fútbol (Garriga, 2007). Así, entonces, en la cultura anglosajona $\mathrm{y}$, en gran medida, en el mundo occidental, toda práctica definida como violenta es concebida como disruptiva, como una ruptura provocada por un elemento no integrado, siempre sorprendente y fuera de tiempo y lugar (Rifiotis y Castelnuovo, 2011).

Para trabajar la posibilidad de aplicar un concepto transcultural de violencia, debe volverse sobre la diferencia etic y emic. Con ella se hace referencia al punto de vista del investigador (etic) y al punto de vista del actor (emic). En el caso concreto de los conceptos relacionados con la violencia, es posible que en el estudio de un grupo cultural o social determinado emerjan conceptos emic que no necesariamente coinciden con definiciones conceptuales propias. A modo de ejemplo, en el caso concreto de estudios sobre violencia en hinchadas de fútbol en Argentina, Garriga (2007) trabaja sobre el concepto del "aguante", que sería "un atributo que distingue a aquellos que pueden en un enfrentamiento corporal ganarse el respeto tanto de los que manejan concepciones diferentes en torno de la violencia como de los iguales" (p. 48). Y el autor dice más adelante: 
El aguante es una forma típica de honor, ya que valora comportamientos y propiedades determinadas como honorables o deshonrosas [...] En la contienda por el aguante vale todo; con esto quiero expresar que no es solo a golpes de puño, sino que también intervienen armas de fuego, navajas, palos, cinturones, piedras y cualquier elemento que pueda ser útil para vencer al rival (p. 49).

Esta concepción emic del aguante contrasta con la visión que los medios de comunicación tienen sobre las prácticas sociales desarrolladas por las hinchadas de fútbol. Este contraste es sumamente atractivo para discutir qué sucede con esas acciones y para comprender que la tarea del investigador social no debe reducirse solamente a explicar las motivaciones internas y el sentido de las prácticas de los actores, sino que debe compararlas y contrastarlas con otras visiones tanto dentro de la misma cultura como fuera de ella.

Debe estudiarse la violencia no solo como una fuerza disruptiva y caótica, sino también como una forma de resolver conflictos y como constructora de lazos sociales (Harris, 1994; Harvey, 1994). De hecho, no todos los conflictos derivan en violencia. En muchos casos, la violencia es consecuencia de una elección entre las posibilidades de resolución existentes, al menos por parte de los victimarios. Por esto, la mayoría de las sociedades tienen estipulaciones y códigos no escritos sobre cómo debe ser ejercida esta violencia. Por ejemplo, en las venganzas de sangre hay prohibiciones ejercidas para que la violencia no escale. Para los nuer del Sudán, entre los miembros de un mismo poblado, las peleas se restringen al uso del garrote y se prohíbe el uso de lanzas. Entre los nuer no se puede abusar de las mujeres ni de los niños, no se pueden destruir chozas ni establos ni tampoco tomar prisioneros. Todas estas restricciones desaparecen cuando los oponentes no son nuer (Riches, 1988; Pritchard, 1977).

\section{Violencia, Biología Y AGRESIÓN}

La discusión sobre el carácter innato de la violencia ha tenido una notable influencia del conductismo. Este afirmaba y acentuaba la base genética de los comportamientos violentos. El término utilizado en estos casos es el de agresión, común no solo al comportamiento humano, sino también al de otros animales. Riches (1988) rescata la definición de Eibl-Eibesfeldt de agresión: "Comportamiento encaminado al espaciamiento de una población (humana o animal) por medio de la repulsión" (p. 41).

Ruiz (2009) hace particular referencia a los estudios que toman en cuenta factores hormonales como productores de agresión: "Un factor biológico que muchos investigadores aseguran que determina la violencia es el hormonal, afirmando que ser varón incrementa el trastorno de personalidad antisocial tres veces más que ser mujer" (p. 4). Estos estudios muestran una mayor agresión masculina que femenina, basados en un más alto porcentaje de testosterona en el hombre. En general, este tipo de investigaciones "biologistas" en las ciencias sociales han producido explicaciones funcionalistas muy básicas. Es evidente que hay un componente biológico como también podemos 
encontrar un componente psicológico, pero la mayoría de las conductas humanas, en cuanto conductas sociales y culturales, se explican en términos sociales y culturales (Riches, 1988, p. 41).

\section{¿Podemos consensuar una DEFINICIÓN TRANSCULTURAL DE VIOLENCIA?}

Estas diferentes concepciones de violencia obligan a volver a la pregunta sobre la posibilidad de adoptar un concepto transcultural de violencia: ¿podemos buscar y acordar un concepto mínimo que nos permita comparar entre una consensuada definición etic y los diversos conceptos emic relacionados? Creo que un acuerdo mínimo transcultural sobre lo que significa la violencia y una ulterior comparación con los significados culturales específicos sería altamente enriquecedor. Poner sobre la mesa el cruzamiento entre las ideas del investigador y las del investigado es uno de los grandes aciertos de la antropología social, que debería ser tenido en cuenta en otras ciencias sociales que estudien estos fenómenos. Las diferencias y los cruces de ambas visiones nos hacen reflexionar y buscar explicaciones novedosas. Una mera descripción del concepto emic sin una referencia a un marco transcultural troncharía nuestra posibilidad de comparar creativamente. En este sentido, Riches habla de un "núcleo universal de significado" del término violencia que podemos acordar para ponerlo en relación con los conceptos culturales específicos.

Una paradoja interesante a la que hace referencia Riches (1988, pp. 18-19) es que el término violencia es utilizado mucho más por las víctimas y los testigos que por los victimarios. Esto permite reflexionar sobre las características negativas que conlleva catalogar a un hecho como violento y a sus ejecutores como violentos. Esa mirada se asienta en el discurso denunciatorio, propio de una lectura criminalizante y estigmatizada contenida en la polaridad víctima-agresor o en la figura jurídica del reo (Rifiotis, 1997). Gran parte de los estudios sobre violencia en ciencias sociales se centran en las víctimas. Wieviorka (2006, p. 241), refiriéndose a la víctima, plantea que la violencia niega la subjetividad del sujeto. Es un terreno ampliamente enriquecedor estudiar no solamente la subjetividad cuestionada de las víctimas (Wieviorka, 2006, p. 241), sino también la subjetividad de los victimarios. Más allá de lo inquietante de sus puntos de vista, ellos tienen mucho que decirnos acerca de sus motivaciones últimas y del sentido social de sus prácticas.

En este punto puede adoptarse una mínima definición válida transcultural de violencia como "una resistida producción de mal físico" (Riches, 1988, p. 28) o, en el mismo sentido, la definición de Marvin (1988): “Toda acción humana que supone una deliberada inflicción de daño hacia otros" (p. 121). Puede usarse alguna de estas dos definiciones, porque, por un lado, son suficientemente amplias para incluir casos que se encuentran en los bordes de la definición de lo violento, como insultos y acosos; $\mathrm{y}$, por otro lado, porque imponen límites en la demarcación de lo que es violento y lo que no lo es. 


\section{EL CONCEPTO DE VIOLENCIA EN EL CONFLICTO COLOMBIANO}

Como ya se ha afirmado, elegir un concepto no es, de ninguna manera, neutral. Puede afirmarse que un fenómeno por analizar es un hecho iluminado por un concepto. Este dará a luz a una serie de elementos aprehensibles de la realidad, y no a otros. Si se hace referencia al conflicto colombiano, se encuentra un caso en donde el Estado-nación parecería incapaz de monopolizar y de legitimar el uso de la violencia o, incluso, de mantener el control sobre la totalidad de su territorio. Diversos actores sociales (la guerrilla, los paramilitares, los narcotraficantes, las fuerzas armadas) luchan violentamente por el control de distintas regiones y territorios colombianos. Como se ha visto, de acuerdo con Riches, para los anglosajones, violencia sería solamente una acción en contra de la ley. Cuando el Estado actúa ejerciendo correctamente su poder de policía, no estaría incurriendo en violencia.

En este sentido, parece obvio entender que, en el caso colombiano, esta asociación entre violencia e ilegitimidad no existe en el sentido local del término. Por un lado, está la sociedad en la que el Estado carece de control efectivo sobre la totalidad del territorio y en la que su poder es desafiado por la acción de múltiples actores sociales (Álvarez, 2004). Por otro lado, la connivencia entre lo paraestatal y lo estatal plantea una deslegitimación del accionar del Estado (Álvarez, 2008). En este contexto, esta visión restrictiva del concepto de violencia asociado a ilegitimidad no es de ninguna utilidad. En cambio, puede considerarse que para un análisis adecuado de la realidad colombiana, es preciso utilizar un concepto transcultural específico de violencia. Así, puede aislarse una serie de prácticas claramente delimitables dentro de las dos definiciones dadas. Utilizar una definición restringida de violencia, como las planteadas por Riches o Marvin, es lo adecuado, ya que deben separarse esos fenómenos específicos de otros. Las estructuras de dominación o las ideas agresivas de masculinidad son elementos que influyen, a veces directa a veces indirectamente, en los comportamientos violentos, pero no son estos comportamientos. Debe arriesgarse, entonces, a privilegiar una definición etic de violencia que permita el desafío de la comparación y de la búsqueda de explicaciones que vayan más allá de una tarea meramente interpretativa que se "muerde su propia cola".

\section{Conclusiones}

Se han reconocido las dificultades que presentan las ciencias sociales para definir a la violencia; el carácter ambiguo y polisémico del término; los intentos de diversos autores por descartar el concepto de plano o, por el contrario, por agrandar y expandir su significado produciendo una verdadera "inflación" de ese. Se descarta, asimismo, una visión "biologista" que interpreta las prácticas violentas como consecuencias de lo biológico y, en particular, de lo genético. En este artículo se propone una interpretación social y cultural a de esas conductas sociales.

Se ha aceptado, también, la posibilidad de desarrollar una definición transcultural de la violencia que permita comparar diversos fenómenos, en diversas culturas y sociedades. En ese sentido, se ha propuesto acordar 
con una mínima definición válida transcultural de violencia, entendiéndola como "una resistida producción de mal físico" (Riches, 1988, p. 28) o como "toda acción humana que supone una deliberada inflicción de daño hacia otros" (Marvin, 1988, p. 121). La utilización de una definición transcultural permite operar en el marco de la comparabilidad y establecer hipótesis explicativas a series similares de fenómenos, pudiendo ir más allá de una mera interpretación de los conceptos emic. Finalmente, se ha discutido la utilización de un concepto transcultural y restringido de violencia para el caso colombiano, acordando que se trataba de la mejor opción. Esta elección permite separar analíticamente esos fenómenos de otros con los que, sin duda, se encuentran conectados e interrelacionados. Pero, dado que estos no poseen las características propias de los fenómenos violentos, un análisis conjunto impediría estudiar a estos últimos en sus especificidades.

\section{REFERENCIAS}

Allison, J. y Kilmartin, C. (2007). Men's violence against women. Theory, research, and activism. Nueva York: Routledge.

Álvarez, S. (2004) Leviatán y sus lobos. Violencia y poder en una comunidad de los Andes colombianos. Buenos Aires: Antropofagia-IDES.

Álvarez, S. (2008). No te bañarás nunca en el mismo río etnográfico. Notas sobre las dificultades del regreso al campo en un pueblo de los Andes colombianos. Estudios en Antropología Social, 1(1). Buenos Aires: Centro de Antropología Social.

Arendt, H. (1969). On violence. Nueva York: Harcourt, Brace, and World.
Benjamin, W. (2010). Crítica de la violencia. Madrid: Biblioteca Nueva.

Bourdieu, P. y Passeron, J. C. (1988). La reproducción. Elementos para una teoría del sistema de enseñanza. México: Fontamara.

Garriga, J. (2007). Haciendo amigos a las piñas. Violencia y redes sociales en una hinchada de fútbol. Bueno Aires: Prometeo.

Garriga, J. y Noel, G. (2010). Notas para una definición antropológica de la violencia: un debate en curso. Publicar en Antropología y Ciencias Sociales, 9, 121-126.

Girard, R. (1985). La violencia y lo sagrado. Barcelona: Anagrama.

Harris, O. (1994). Condor and Bull. The ambiguities of masculinity in Northern Potosi. En P. Harvey \& P. Gow. Sex and violence. Issues in representation and experience. Nueva York: Routledge.

Harvey, P. (1994). Domestic violence in the Peruvian Andes. En P. Harvey \& P. Gow. Sex and violence. Issues in representation and experience. Nueva York: Routledge.

Heelas, P. (1989). Anthropology, violence and catharsis. En P. Marsh \& A. Campbell. Agression and violence. Londres: Basil and Blackwell.

Kron-Hansen, C. (1994). The anthropology of violent Interaction. Journal of Anthropological Research, 50(4), 367-381.

Marvin, G. (1988). Honor, integridad y el problema de la violencia en la corrida de toros en España. En D. Riches. El fenómeno de la violencia. Madrid: Pirámide. .

Moore, H. (1994). The problem of explaining violence. En P. Harvey \& P. Gow. Sex and violence. Issues in representation and experience. Nueva York: Routledge.

Pritchard, E. (1977). Los nuer. Barcelona: Anagrama.

Riches, D. (1988). El fenómeno de la violencia. Madrid: Pirámide. 
Rifiotis, T. (1997). Nos campos da violencia: deferença e positividade. Antropología em Primeira Mão, 19. Porto Alegre.

Rifiotis, T. y Castelnuovo, N. (2011). La violencia como punto de partida. En Antropología, violencia y justicia. Repensando matrices de sociabilidad contemporánea en el campo del género y de la família. Buenos Aires: Antropofagia.

Ruiz, Y. (2009). Biología, cultura y violencia. Presentación realizada para las XIV Jornades de Foment de la investigació. Barcelona: Facultat de Ciénces Humanes i Socials, Universidad Jaume I.
Taussig, M. (1984). Culture of terror, space of death: Roger Casement's Putumayo report and the explanation of torture. Comparative Studies in Society and History, 26, 467-497. Cambridge.

Taussig, M. (1986). Shamanism, Colonialism, and the Wild man. A study in terror and healing. Chicago: University of Chicago Press.

Taussig, M. (1999). Maleficium: el fetichismo del Estado. En Un gigante en convulsiones. El mundo humano como sistema nervioso en emergencia permanente. Barcelona: Gedisa.

Wieviorka, M. (2006, enero-junio). La violencia: destrucción y constitución del sujeto. Espacio Abierto, Cuaderno Venezolano de Sociología, 15, 239-248. Universidad de Zulia. 